\title{
ALGEBRAIC CORRESPONDENCES BETWEEN GENUS THREE CURVES AND CERTAIN CALABI-YAU VARIETIES
}

\author{
TOMOHIDE TERASOMA
}

\section{INTRODUCTION}

Let $\Phi_{L}: \mathbf{P}^{3}--\rightarrow \mathbf{P}\left(L^{*}\right)$ be a net of quadrics, i.e. two dimensional linear system of quadrics. If the linear system $\Phi_{L}$ is sufficiently generic, then

(1) the base locus $B$ consists of eight points $b_{1}, \ldots, b_{8}$, and

(2) the locus $C$ of singular quadrics in $\mathbf{P}(L)$ is a smooth plane quartic curve.

A set $B$ of eight points in $\mathbf{P}^{3}$ of this form is called a regular Cayley octad and the plane quartic curve $C$ is called the associated curve of $B$. The moduli space of regular Cayley octads is isomorphic to the moduli space of plane quartic curves with markings of even theta divisors (cf. [DO]).

Let $B=\left\{b_{1}, \ldots, b_{8}\right\}$ be a regular Cayley octad, $H_{1}, \ldots, H_{8}$ hyperplanes in the dual projective space $\widehat{\mathbf{P}^{3}}=\left\{\left(\xi_{1}: \xi_{2}: \xi_{3}: \xi_{4}\right)\right\}$ of $\mathbf{P}^{3}$ corresponding to the points $b_{1}, \ldots, b_{8}$, and $X$ the double covering of $\widehat{\mathbf{P}^{3}}$ branched along the eight hyperplanes $H_{1}, \ldots, H_{8}$. In this paper, we study a certain algebraic correspondence between the associated curve $C$ and the double covering $X$ of $\widehat{\mathbf{P}^{3}}$ based on a geometry of curves on $X$. Since the variety $X$ has only quotient singularities, $H^{i}(X, \mathbf{Q})$ is equipped with a pure Hodge structure of weight $i$. The first main theorem of this paper is the following.

Theorem 1.1 (Theorem 5.1). Under the above notations, we have an injection of Hodge structures

$$
\text { cyl }: H^{1}(C, \mathbf{Q})(-1) \rightarrow H^{3}(X, \mathbf{Q})
$$

induced by an algebraic correspondence. In particular, the Hodge structure $H^{3}(X, \mathbf{Q})$ is not irreducible.

The same algebraic correspondence is constructed independently in unpublished work by I.Dolgachev-E.Merkmann (1994). The author would like to express his thanks to Prof. I.Dolgachev for the communication. We remark that the above homomorphism specializes to that defined by the "symmetric construction" in [T1], if the curve $C$ specializes to a hyperelliptic curve. Let us recall the symmetric construction briefly. Let $C$ be a hyperelliptic curve of genus three defined by

$$
y^{2}=\prod_{i=1}^{8}\left(x-\lambda_{i}\right)
$$


We define a double covering $X$ of $\widehat{\mathbf{P}^{3}}$ by

$$
\eta^{2}=\prod_{i=1}^{8}\left(\lambda_{i}^{3} \xi_{1}+\lambda_{i}^{2} \xi_{2}+\lambda_{i} \xi_{3}+\xi_{4}\right)
$$

in the weighted projective space $\mathbf{P}(1,1,1,1,4)$. Here $\xi_{0}, \xi_{1}, \xi_{2}, \xi_{3}$ are homogeneous coordinates of degree one and $\eta$ is that of degree four. Then the variety $X$ is a Calabi-Yau variety, i.e. it has only Gorenstein singularities and has the trivial dualizing sheaf admitting a global crepant resolution (see [CM]).

Let $C^{(1)}, C^{(2)}, C^{(3)}$ be three copies of the curve $C$. The action of $\mu_{2}=\langle\iota\rangle$ on $C$ defined by the hyperelliptic involution $\iota$ yields an action of $\mu_{2}{ }^{3}$ on $C^{3}=C^{(1)} \times C^{(2)} \times C^{(3)}$. The symmetric group $\mathfrak{S}_{3}$ of degree three acts on $C^{3}$ by permuting the components, and as a consequence, we obtain an action of semi-direct product $\left(\mu_{2}{ }^{3}\right) \rtimes \mathfrak{S}_{3}$ on $C^{3}$. Let $N$ be the kernel of the homomorphism $\mu_{2}{ }^{3} \rightarrow \mu_{2}:\left(\zeta_{1}, \zeta_{2}, \zeta_{3}\right) \mapsto \zeta_{1} \zeta_{2} \zeta_{3}$. Then the semi-direct product $G=N \rtimes \mathfrak{S}_{3}$ is a normal subgroup of $\mu_{2}{ }^{3} \rtimes \mathfrak{S}_{3}$ of index two.

Since the quotient of $C^{3}$ by the group $\left(\mu_{2}{ }^{3}\right) \rtimes \mathfrak{S}_{3}$ is isomorphic to $\left(\mathbf{P}^{1}\right)^{3} / \mathfrak{S}_{3} \simeq$ $\mathbf{P}^{3}$, the quotient of $C^{3}$ by $G$ is a double covering of $\mathbf{P}^{3}$. Since the covering map

$$
C^{3} / G \rightarrow C^{3} /\left(\mu_{2}^{3}\right) \rtimes \mathfrak{S}_{3} \simeq \mathbf{P}^{3}
$$

branches exactly along the eight hyperplanes $H_{i}=\left\{\lambda_{i}^{3} \xi_{1}+\lambda_{i}^{2} \xi_{2}+\lambda_{i} \xi_{3}+\xi_{4}=\right.$ $0\}$, we have an isomorphism

$$
X \stackrel{\sim}{\rightarrow} C^{3} / G
$$

over $\left(\mathbf{P}^{1}\right)^{3} / \mathfrak{S}_{3} \simeq \mathbf{P}^{3}$. The above isomorphism is written as

$$
\begin{aligned}
& \left(\xi_{1}: \xi_{2}: \xi_{3}: \xi_{4}: \eta\right) \\
& =\left(-1: x_{1}+x_{2}+x_{3}:-\left(x_{1} x_{2}+x_{2} x_{3}+x_{3} x_{1}\right): x_{1} x_{2} x_{3}: y_{1} y_{2} y_{3}\right) .
\end{aligned}
$$

on the affine part of $C^{3}$. Here $\left(x_{i}, y_{i}\right)$ is a coordinate of the affine part of $C^{(i)}$ for $i=1,2,3$.

Since the variety $X$ has only rational singularities, $H^{3}(X, \mathbf{Q})$ is equipped with a pure Hodge structure of weight three. By the isomorphism (1.2), we have an isomorphism of Hodge structures :

$$
\bigwedge^{3} H^{1}(C, \mathbf{Q}) \simeq H^{3}(X, \mathbf{Q})
$$

The composite morphism

$$
\begin{array}{ccccc}
c y l: & C^{(1)} \times C & \rightarrow & C^{(1)} \times C^{(2)} \times C^{(3)} & \stackrel{\varphi}{\rightarrow} \quad X \\
p_{1} \times p & \mapsto & p_{1} \times \iota\left(p_{1}\right) \times p
\end{array}
$$

defines a family of smooth rational curves in $X$ parameterized by $C$. (See Section 4.3 for details.) From this family of smooth rational curves in $X$, we get a homomorphism of Hodge structures:

$$
\text { cyl }: H^{1}(C, \mathbf{Q})(-1) \rightarrow H^{3}(X, \mathbf{Q}) .
$$

This map is the specialization of the map (1.1) in the following sense. Let $M$ be the moduli space of genus three curves with level $2 N$-structures $(N \geq 2)$ 
and $p: \mathcal{C} \rightarrow M$ and $\Xi: \mathcal{X} \rightarrow M$ the universal families of plane quartic curves and double coverings of $\mathbf{P}^{3}$. The family of algebraic correspondences in Theorem 1.1 give rise to a homomorphism of variations of Hodge structures:

$$
c y l: R^{1} p_{*} \mathbf{Q}(-1) \rightarrow R^{3} \Xi_{*} \mathbf{Q} .
$$

The variations of Hodge structures $R^{1} p_{*} \mathbf{Q}(-1)$ and $\mathbf{R}^{3} \Xi_{*} \mathbf{Q}$ and the homomorphism cyl extend to those on $M$. The homomorphism (1.4) is the fiber of (1.5) at the point corresponding to the hyperelliptic curve $C$.

In the last part of this paper, we study the variation of Hodge structures obtained by the cokernel Coker $(c y l)$ of the homomorphism (1.5). By the isomorphism (1.3), the restriction of $\operatorname{Coker}(c y l)$ to the hyperelliptic locus is isomorphic to the primitive part of the third higher direct image of the relative Jacobian scheme as variations of Hodge structures. The Hodge type of Coker $(c y l)$ at each fiber is $(1,6,6,1)$. Nevertheless, we have the following theorem.

Theorem 1.2 (Theorem 6.6). There exists no polarized abelian scheme a: $\mathcal{A} \rightarrow M$ of relative dimension three, whose degree three primitive part

$$
R^{3} a_{*, \operatorname{prim}} \mathbf{Q}=\operatorname{Coker}\left(R^{1} a_{*} \mathbf{Q}(-1) \stackrel{L}{\rightarrow} R^{3} a_{*} \mathbf{Q}\right)
$$

of the higher direct image is isomorphic to Coker(cyl). Here L denotes the Lefschetz operator for the polarization.

The contents of the paper is as follows. We recall several basic facts concerning net of quadrics in Section 2. In Section 3, we construct an algebraic correspondence between a non-hyperelliptic curve of genus three and a double covering of $\widehat{\mathbf{P}^{3}}$ using the theory of net of quadrics. In Section 4 and 5, we prove the injectivity of the homomorphism induced by this correspondence (Theorem 1.1). In Section 6 and Section 7, we compute infinitesimal variations of Hodge structure to conclude Theorem 1.2 .

Acknowledgment. The author would like to thank Keiji Matsumoto for discussions, which gives a motivation of this paper. The statement of Theorem 1.2 is a refinement of the first version of this paper, which is suggested by the referee. The author would like to express his thanks to the referee. After finishing this paper, the relevant paper GSZ was pointed out by K.Zuo and B.van Geemen, which treats general configurations of eight hyperplanes and hyperelliptic locus. The author is grateful for the information.

Notation 1.3. Quadratic polynomials $Q\left(x_{1}, \ldots, x_{k}\right)$ of $x_{1}, \ldots, x_{k}$ are in one to one correspondence with $k \times k$ symmetric matrices $Q^{\prime}$ by the relation $Q\left(x_{1}, \ldots, x_{k}\right)=\left(x_{1}, \ldots, x_{k}\right) Q^{\prime t}\left(x_{1}, \ldots, x_{k}\right)$. The corresponding symmetric matrix $Q^{\prime}$ is also denoted by $Q$ if there are no confusions. The rank of the quadric is defined by the rank of the corresponding symmetric matrix. The space of quadratic form on $V$ is denoted by $\operatorname{Sym}^{2}(V)$.

For a vector space $V$, the projective space $(V-\{0\}) / \mathbf{C}^{\times}$associated to $V$ is denoted as $\mathbf{P}(V)$. For a homogeneous polynomial $f$ on $V$, the subvariety of $\mathbf{P}(V)$ defined by $f$ is denoted as $Z(f)$. The point of the dual projective 
space $\mathbf{P}\left(V^{*}\right)$ corresponding to a hyperplane $W \subset \mathbf{P}(V)$ is denoted by $[W]$. The hyperplane in $\mathbf{P}\left(V^{*}\right)$ associated to a point $b \in \mathbf{P}(V)$ is denoted by $M_{b}$.

\section{Net of quadrics in the three Dimensional PROJECTIVE SPACE}

Let $V$ be a four dimensional vector space. For a three dimensional subspace $L$ in $\operatorname{Sym}^{2}(V)$, the associated linear system $\mathbf{P}(V)--\rightarrow \mathbf{P}\left(L^{*}\right)$ is denoted as $\Phi_{L}$. A two dimensional linear system is called a net. The member of the linear system corresponding to $t \in \mathbf{P}(L)$ is denoted as $Q_{t} \subset \mathbf{P}(V)$. The locus of singular quadrics in $\mathbf{P}(L)$ is denoted as $C(L)$. A net of quadrics in $\mathbf{P}(V)$ is called regular if $C(L)$ is smooth. In this case, $C(L)$ is a smooth plane quartic curve and is called the plane quartic curve associated to $L$. By choosing a basis $Q_{1}, Q_{2}, Q_{3}$ of $L$ and basis $x_{0}, \ldots, x_{3}$ of $V$, the subvariety $C(L)$ of $\mathbf{P}(L)$ is defined by the polynomial $\operatorname{det}\left(t_{1} Q_{1}+t_{2} Q_{2}+t_{3} Q_{3}\right)$. For a regular net of quadrics $L$, the rank of the quadric $t_{1} Q_{1}+t_{2} Q_{2}+t_{3} Q_{3}$ is three for all $t=\left(t_{1}: t_{2}: t_{3}\right) \in C(L)$ and the base locus $B$ of the net of quadrics $\Phi_{L}$ consists of distinct eight points. Moreover any four points in the base locus do not lie on a common plane.

Let $b_{1}, \ldots, b_{7}$ be generic seven points in $\mathbf{P}(V)$. Then the space $L$ of quadratic polynomials on $V$ vanishing at $b_{1}, \ldots, b_{7}$ is a three dimensional vector space, and $\Phi_{L}$ is a regular net of quadrics. Moreover the base locus $B$ of the net of quadrics is a zero dimensional reduced subscheme consisting of eight points containing $\left\{b_{1}, \ldots, b_{7}\right\}$.

A configuration of eight points in $\mathbf{P}(V)$ is called a regular Cayley octad if it is the base locus of a regular net of quadrics. By associating the base locus a net of quadrics, we get a one to one correspondence between the set of regular nets of quadrics in $\mathbf{P}(V)$ and that of regular Cayley octads. The set of choices of two points in $\left\{b_{1}, \ldots, b_{8}\right\}$ corresponds one to one to the set of odd theta divisors of $C(L)$, and there is one to one correspondence between the set of odd theta divisors and that of bitangents of $C(L)$.

We have the following four moduli spaces.

(1) The moduli space $M_{1}$ of regular Cayley octads $\left\{b_{1}, \ldots, b_{8}\right\}$ in $\mathbf{P}(V)$.

(2) The moduli space $M_{2}$ of regular nets of quadrics in $\mathbf{P}(V)$.

(3) The moduli space $M_{3}$ of smooth plane quartic curves.

(4) The moduli space $M_{4}$ of non-hyperelliptic smooth curves of genus three.

Then we have the following morphisms

$$
M_{1} \stackrel{f_{1}}{\rightarrow} M_{2} \stackrel{f_{2}}{\rightarrow} M_{3} \stackrel{f_{3}}{\rightarrow} M_{4}
$$

The morphisms $f_{1}$ and $f_{3}$ are isomorphisms and the morphism $f_{2}$ is an etale finite morphism of degree 36 .

Definition 2.1 (Steinerian curve). Let $\left\langle Q_{1}, Q_{2}, Q_{3}\right\rangle$ be a regular net of quadrics and $C$ the associated plane quartic curve. Then $Z\left(Q_{t}\right)$ is a cone over a smooth conic in $\mathbf{P}(V)$ for $t \in C$. By attaching the vertex $s(t)$ of $Z\left(Q_{t}\right)$ to a point 
$t \in C$, we have a map $s: C \rightarrow \mathbf{P}(V)$. The image $s(C)$ of $C$ under the morphism $s$ is called the Steinerian curve ([DO]).

It is known that the Steinerian curve is of degree 6 and isomorphic to $C$ via the map $s: C \rightarrow \mathbf{P}(V)$. The Steinerian curve is disjoint from the base locus of the net of quadrics. The embedding $s$ is equal to the linear system defined by the sum of canonical class and one of 36 even theta divisors.

\section{Double COVERIng BRANCHED AlOng EIGHT HyPERPlanes}

We use the same notations as in the last section. Let $\left\{b_{1}, \ldots, b_{8}\right\}$ be a regular Cayley octad in $\mathbf{P}(V)$ and $C$ be the associated plane curve. Let $H_{1}, \ldots, H_{8}$ be the hyperplanes corresponding to $b_{1}, \ldots, b_{8}$ in the dual projective space $\mathbf{P}\left(V^{*}\right)$ of $\mathbf{P}(V)$. Let $X$ be the double covering branched along the union of the hyperplanes $H_{1}, \ldots, H_{8}$. The covering map $X \rightarrow \mathbf{P}\left(V^{*}\right)$ is denoted by $\pi$. Since any four points in $\left\{b_{1}, \ldots, b_{8}\right\}$ are not contained in a common plane by the condition of regular Cayley octad, the union $D=\cup_{i} H_{i}$ is a normal crossing divisor. Therefore the double covering $X$ has only Gorenstein singularities and the trivial dualizing sheaf admitting a global crepant resolution (see $[\mathrm{CM}]$ ). A variety with this property is called a Calabi-Yau variety.

In this section, we define a double covering $C^{\#}$ of the curve $C$ and a closed subvariety $U^{\#}$ in $C^{\#} \times X$ of codimension two. A component $U$ of $U^{\#}$ defines an algebraic correspondence between the varieties $C$ and $X$.

Let $t$ be a point in $C$. Then $Q_{t} \subset \mathbf{P}(V)$ is a cone over a smooth conic. We define a rational curve $\gamma_{t}$ in $\mathbf{P}\left(V^{*}\right)$ by

$$
\gamma_{t}=\left\{[W] \in \mathbf{P}\left(V^{*}\right) \mid W \text { is tangent to the cone } Q_{t}\right\} .
$$

If $[W] \in \gamma_{t}$, then $W$ contains a vertex $s(t) \in \mathbf{P}(V)$. Therefore the rational curve $\gamma_{t}$ is contained in the hyperplane $M_{s(t)}$. (For the notation $M_{*}$ and [*], see Notation 1.3.)

Proposition 3.1. The curve $\gamma_{t}$ is tangent to the hyperplanes $H_{1}, \ldots, H_{8}$ for all $t \in C$.

Proof. The quadric $Q_{t}$ contains any of the base points $b_{i}$. Since the linear system $\Phi_{L}$ is regular, $b_{i}$ does not coincide with $s(t)$ for any $t \in C$. Therefore the conic $Q_{t}$ is smooth at the point $b_{i}$ and there is only one tangent hyperplane of $Q_{t}$ containing $b_{i}$. Therefore the hyperplane $H_{i}=M_{b_{i}}$ is tangent to the conic $\gamma_{i}$. Thus we have proved the proposition.

Corollary 3.2. Under the assumption of Proposition 3.1, the inverse image $\pi^{-1}\left(\gamma_{t}\right)$ is the union of two rational curves $\tilde{\gamma}_{t}^{(1)}$ and $\tilde{\gamma}_{t}^{(2)}$.

Proof. By Proposition [3.1, the normalization of $\pi^{-1}\left(\gamma_{t}\right)$ is an etale double covering of a smooth rational curve. Therefore it is a disjoint union of two copies of $\gamma_{t}$. 
By attaching the irreducible components of $\pi^{-1}\left(\gamma_{t}\right)$ to $t \in C$, we get a double covering $C^{\#}$ (possibly not irreducible) of $C$. More precisely, the covering $C^{\#}$ is defined as the Stein factorization of the normalization of the map

$$
\left\{(x, t) \in X \times C \mid \pi(x) \in \gamma_{t}\right\} \rightarrow C
$$

obtained by the second projection. The irreducible component of $\pi^{-1}\left(\gamma_{t}\right)$ corresponding to a point $\tilde{t} \in C^{\#}$ is written as $\tilde{\gamma}_{\tilde{t}}$.

Lemma 3.3. The covering $C^{\#}$ is etale over $C$.

Proof. Since the rank of $Q_{t}$ is equal to 3 for any point $t \in C, \gamma_{t}$ is a smooth rational curve of degree two in $\mathbf{P}\left(V^{*}\right)$. We will show that it is not contained in $H_{i}$ for any $i$. The curve $\gamma_{t}$ is contained in $H_{i}$ if and only if the vertex $s(t)$ of the quadric $Q_{t}$ coincides with one of a base point $b_{i}$ but for a smooth plane curve $C$ of degree four, any intersection of bitangents is not contained in $C$. Therefore $\gamma_{t}$ is not contained in $H_{i}$. As a consequence, $\pi^{-1}\left(\gamma_{t}\right)$ has exactly two irreducible components and $C^{\#}$ is etale over $C$.

Let $U^{\#}$ be the universal family

$$
U^{\#}=\left\{(\tilde{t}, x) \in C^{\#} \times X \mid x \in \tilde{\gamma}_{\tilde{t}}\right\} .
$$

Then we have the following diagram:

$$
\begin{aligned}
& U^{\#} \subset C^{\#} \times X \stackrel{p r_{2}}{\rightarrow} X \\
& \begin{array}{c}
p r_{1} \downarrow \\
C^{\#} .
\end{array}
\end{aligned}
$$

Proposition 3.4. The curve $C^{\#}$ is a union of two copies $C^{(1)}$ and $C^{(2)}$ of $C$.

Proof. To prove the proposition, it is enough to prove the monodromy action on the irreducible components of $\pi^{-1}\left(\gamma_{t}\right)$ is trivial. We compute the monodromy action when the plane quartic curve tends to a hyperelliptic curve. This will be done in Proposition 4.4.

Definition 3.5 (Algebraic correspondence $\mathcal{U}$ ). We define $U \subset C \times X$ by the pull back of $U^{\#}$ by the map $C \times X \stackrel{\simeq}{\rightarrow} C^{(1)} \times X \subset C^{\#} \times X$.

\section{TWISTED CUBIC AND HYPERELLIPTIC CURVES}

4.1. Special net of quadrics. In this subsection, we consider a special net of quadrics generated by

$$
Q_{1}=\left(\begin{array}{cccc}
0 & 0 & 1 & 0 \\
0 & -2 & 0 & 0 \\
1 & 0 & 0 & 0 \\
0 & 0 & 0 & 0
\end{array}\right), Q_{2}=\left(\begin{array}{cccc}
0 & 0 & 0 & -1 \\
0 & 0 & 1 & 0 \\
0 & 1 & 0 & 0 \\
-1 & 0 & 0 & 0
\end{array}\right), Q_{3}=\left(\begin{array}{cccc}
0 & 0 & 0 & 0 \\
0 & 0 & 0 & 1 \\
0 & 0 & -2 & 0 \\
0 & 1 & 0 & 0
\end{array}\right) .
$$


Here we used the correspondence between quadratic polynomials and symmetric matrices in Notation 1.3. Then the intersection $T=Z\left(Q_{1}\right) \cap Z\left(Q_{2}\right) \cap Z\left(Q_{3}\right)$ is a twisted cubic curve defined by the image of the map

$$
s: \mathbf{P}^{1} \rightarrow \mathbf{P}(V): x \mapsto\left(x^{3}: x^{2}: x: 1\right) .
$$

The defining equation of the singular locus of the net of quadrics $\Phi_{\left\langle Q_{1}, Q_{2}, Q_{3}\right\rangle}$ is equal to

$$
\operatorname{det}\left(t_{1} Q_{1}+t_{2} Q_{2}+t_{3} Q_{3}\right)=\left(t_{1} t_{3}-t_{2}^{2}\right)^{2}=0 .
$$

The conic $\left\{t_{1} t_{3}-t_{2}^{2}=0\right\}$ in $\mathbf{P}(L)$ is denoted as $D$. We choose a parameter of $D$ as $\left(t_{1}: t_{2}: t_{3}\right)=\left(1: x: x^{2}\right)$. The singular quadratic polynomial $Q_{t}$ for $t=\left(t_{1}: t_{2}: t_{3}\right)=\left(1: x: x^{2}\right) \in D$ is written as $Q(x)$.

Lemma 4.1. For $\left(1: x: x^{2}\right) \in D$, we define a curve $\gamma_{x}$ in $\mathbf{P}\left(V^{*}\right)$ by

$$
\gamma_{x}=\left\{[W] \in \mathbf{P}\left(V^{*}\right) \mid W \text { is tangent to } Z(Q(x))\right\} .
$$

Then $\gamma_{x}$ is equal to

$$
\gamma_{x}=\left\{\left(\xi_{1}: \xi_{2}: \xi_{3}: \xi_{4}\right)=\left(-1: 2 w+x:-\left(w^{2}+2 x w\right): w^{2} x\right) \mid w \in \mathbf{P}^{1}\right\} .
$$

Moreover, the vertex $v(x)$ of $V(Q(x))$ is

$$
\left(p_{1}: p_{2}: p_{3}: p_{4}\right)=s(x)=\left(x^{3}: x^{2}: x: 1\right) .
$$

Proof. By direct computation, we can check that the hyperplane

$$
\begin{aligned}
W_{w}=\{ & \left(p_{1}: p_{2}: p_{3}: p_{4}\right) \in \mathbf{P}(V) \mid \\
& \left.-p_{1}+(2 w+x) p_{2}-\left(w^{2}+2 x w\right) p_{3}+w^{2} x p_{4}=0\right\}
\end{aligned}
$$

is tangent to the quadric $Z(Q(x))=\left\{Q(x)=\left(p_{1} p_{3}-p_{2}^{2}\right)+x\left(p_{2} p_{3}-p_{1} p_{4}\right)+\right.$ $\left.x^{2}\left(p_{2} p_{4}-p_{3}^{2}\right)=0\right\}$. Since the curve $\gamma_{x}$ is known to be of degree two, we have proved the lemma.

4.2. A deformation of special net of quadrics. Let $\Delta(u)=\{u \in \mathbf{C} \mid$ $|u|<\epsilon\}$ be a sufficiently small disk. The punctured dis $\Delta(u)-\{0\}$ is denoted by $\Delta^{*}(u)$.

Proposition 4.2. Let $Q_{1}, Q_{2}$ and $Q_{3}$ be the quadrics defined in (4.1).

Let $\left\langle Q_{1}(u), Q_{2}(u), Q_{3}(u)\right\rangle$ be a generic deformation over $\Delta(u)$ of $L=$ $\left\langle Q_{1}, Q_{2}, Q_{3}\right\rangle$ such that $Q_{i}(0)=Q_{i}$. Then the equation of the discriminant locus $F\left(t_{1}, t_{2}, t_{3}, u\right)=\operatorname{det}\left(t_{1} Q_{1}(u)+t_{2} Q_{2}(u)+t_{3} Q_{3}(u)\right)$ can be written as

$$
F\left(t_{1}, t_{2}, t_{3}, u\right) \equiv\left(t_{1} t_{3}-t_{2}^{2}\right)^{2}+u f\left(t_{1}, t_{2}, t_{3}\right) \quad\left(\bmod u^{2} \mathbf{C}\left[t_{1}, t_{2}, t_{3}, u\right]\right)
$$

such that $\left\{f\left(t_{1}, t_{2}, t_{3}\right)\right\} \cap D$ consists of distinct eight points and $C^{\prime}:\left\{f\left(t_{1}, t_{2}, t_{3}\right)=\right.$ $0\}$ is a smooth plane curve. 
Proof. We consider a deformation given by

$Q_{1}(u)=\left(\begin{array}{cccc}0 & 0 & 1 & 0 \\ 0 & -2 & 0 & 0 \\ 1 & 0 & 0 & 0 \\ 0 & 0 & 0 & 4 u\end{array}\right), \quad Q_{2}(u)=\left(\begin{array}{cccc}0 & 0 & 0 & -u-1 \\ 0 & 0 & 3 u+1 & 0 \\ 0 & 3 u+1 & 0 & 0 \\ -u-1 & 0 & 0 & 0\end{array}\right)$,

$Q_{3}(u)=\left(\begin{array}{cccc}4 u & 0 & 0 & 0 \\ 0 & 0 & 0 & 1 \\ 0 & 0 & -2 & 0 \\ 0 & 1 & 0 & 0\end{array}\right)$.

Then we have $Q_{i}(0)=Q_{i}$ for $i=1,2,3$, and

$$
\begin{array}{r}
\operatorname{det}\left(t_{1} Q_{1}(u)+t_{2} Q_{2}(u)+t_{3} Q_{3}(u)\right) \equiv\left(t_{1} t_{3}-t_{2}^{2}\right)^{2}+8\left(t_{1}^{4}+t_{2}^{4}+t_{3}^{4}\right) u \\
\left(\bmod u^{2} \mathbf{C}\left[t_{1}, t_{2}, t_{3}, u\right]\right)
\end{array}
$$

The intersection of $C^{\prime}=\left\{8\left(t_{1}^{4}+t_{2}^{4}+t_{3}^{4}\right)=0\right\}$ and $D$ consists of distinct eight points and $C^{\prime}$ is smooth. Thus we have proved the proposition for a generic deformation.

4.3. Smooth family $\tilde{\mathcal{C}}$ and its double covering $\mathcal{C}^{\#}$. Let $f\left(t_{1}, t_{2}, t_{3}\right)$ be a generic homogeneous polynomial of degree four. Then the zero locus $Z(f)$ is smooth and the set $\left\{\left(t_{1}: t_{2}: t_{3}\right) \mid t_{1} t_{3}-t_{2}^{2}=0, f\left(t_{1}, t_{2}, t_{3}\right)=0\right\}$ consists of distinct eight points

$$
\tau_{i}=\left(1: \lambda_{i}: \lambda_{i}^{2}\right) \quad(i=1, \ldots, 8) .
$$

We define a family of plane curves $\mathcal{C}$ of degree four over $\Delta(u)$ by

$$
\mathcal{C}=\left\{\left(u,\left(t_{1}: t_{2}: t_{3}\right) \mid\left(t_{1} t_{3}-t_{2}^{2}\right)^{2}-u f\left(t_{1}, t_{2}, t_{3}\right)\right\}\right.
$$

By Proposition 4.2, there is a family of nets of quadrics $\left\langle Q_{1}(u), Q_{2}(u), Q_{3}(u)\right\rangle$ in $\mathbf{P}(V)$ such that the family of associated plane quartic curve is isomorphic to $\mathcal{C}$.

By changing the base $\Delta(v) \rightarrow \Delta(u)$ defined by $v \mapsto v^{2}=u$, we have a family $\mathcal{C} \times_{\Delta(u)} \Delta(v)$ of plane curve in $\mathbf{P}(L) \times \Delta(v)$ over $\Delta(v)$. Then the normalization $\tilde{\mathcal{C}}$ of $\mathcal{C} \times_{\Delta(u)} \Delta(v)$ is isomorphic to

$$
\left\{\left(v,\left(\nu: t_{1}: t_{2}: t_{3}\right)\right) \in \Delta(v) \times \mathbf{P}(2,1,1,1) \mid t_{1} t_{3}-t_{2}^{2}=\nu v, \nu^{2}=f\left(t_{1}, t_{2}, t_{3}\right)\right\},
$$

where $\left(\nu: t_{1}: t_{2}: t_{3}\right)$ is the coordinates of the weighted projective space $\mathbf{P}(2,1,1,1)$. Thus we have a smooth family

$$
p: \tilde{\mathcal{C}} \rightarrow \Delta(v)
$$

of curves of genus three over $\Delta(v)$. The central fiber $p^{-1}(0)=\tilde{\mathcal{C}}_{0}$ is a hyperelliptic curve. The inverse image $p^{-1}\left(\Delta(v)^{*}\right)$ of $\Delta(v)^{*}$ is denoted as $\tilde{\mathcal{C}^{0}}$.

We have a family of regular Cayley octads

$$
\beta_{0}: \mathcal{B}^{0} \rightarrow \mathbf{P}(V) \times \Delta^{*}(v) \rightarrow \Delta^{*}(v)
$$

of $\tilde{\mathcal{C}^{0}} \rightarrow \Delta^{*}(v)$ over $\Delta^{*}(v)$ and its closure in $\mathbf{P}(V) \times \Delta(v)$ is denoted as $\beta: \mathcal{B} \rightarrow \Delta(v)$. 
Proposition 4.3. (1) Let $v_{0} \neq 0$ be an element in $\Delta(v)$. Then the monodromy substitution on $\mathcal{B}_{v_{0}}^{0}=\left\{b_{1}\left(v_{0}\right), \ldots, b_{8}\left(v_{0}\right)\right\}$ around $v=0$ is trivial. Especially $b_{i}\left(v_{0}\right)$ extends to a section $b_{i}(v)$ to $\mathbf{P}(V)$ over $\Delta(v)$.

(2) The point $b_{i}(v)$ converges to the point $s\left(\lambda_{i}\right)$ when $v$ tends to zero.

Proof. (1) Choose two elements $b_{i}\left(v_{0}\right)$ and $b_{j}\left(v_{0}\right)$. The line connecting them in $\mathbf{P}(V) \times\left\{v_{0}\right\}$ is denoted as $l_{i j}\left(v_{0}\right)$. There are 28 lines of such form. Then $l_{i j}\left(v_{0}\right)$ intersect the Steinerian curve at two points $p_{i j}^{(1)}\left(v_{0}\right), p_{i j}^{(2)}\left(v_{0}\right)$. The intersection points correspond to the bitangent points of the plane quartic curve $\tilde{\mathcal{C}_{v_{0}}}$ in $\mathbf{P}(L)$. The divisor $p_{i j}^{(1)}\left(v_{0}\right)+p_{i j}^{(2)}\left(v_{0}\right)$ is one of 28 odd theta divisors. Since the family of curves $\tilde{\mathcal{C}}$ is smooth over $\Delta(v)$, the monodromy action on the 28 lines $l_{i j}\left(v_{0}\right)$ is trivial. Therefore the monodromy action on the set $\left\{b_{i}\left(v_{0}\right)\right\}$ is trivial.

(2) The curve $\tilde{\mathcal{C}_{0}}$ is a hyperelliptic curve which is the double covering of $D$ branched at $\tau_{1}, \ldots, \tau_{8}$. Since the base locus of $\left\langle Q_{1}(0), Q_{2}(0), Q_{3}(0)\right\rangle$ is equal to $D$, we have $\left\{\tau_{1}, \ldots, \tau_{8}\right\} \subset D$. For a hyperelliptic curve $\tilde{\mathcal{C}}_{0}$, an odd theta divisor is obtained by taking sum of distinct two points in the branch points. Therefore the family of lines $l_{i j}(v)$ connecting $b_{i}(v)$ and $b_{j}(v)$ converges to the line connecting the image $s\left(\lambda_{i}\right)$ and $s\left(\lambda_{j}\right)$. Therefore $b_{i}(v)$ converges to $s\left(\lambda_{i}\right)$.

By the above proposition, the fiber $\beta^{-1}(0)$ of $\beta$ at 0 consists of distinct 8 points $\left\{\left(\lambda_{i}^{3}: \lambda_{i}^{2}: \lambda_{i}: 1\right)\right\}_{i=1, \ldots, 8}$. We chose a lifting $\tilde{b}_{i}(v)=\left(b_{1 i}, \cdots, b_{4 i}\right)$ of the section $b_{i}(v)$ from $\Delta(v)$ to $V-\{0\}$ such that $\tilde{b}_{i}(0)=\left(\lambda_{i}^{3}, \lambda_{i}^{2}, \lambda_{i}, 1\right)$. We consider a family of Calabi-Yau varieties $\mathcal{X}$ over $\Delta(v)$ defined by

$$
\mathcal{X}_{v}: \eta^{2}=\prod_{i=1}^{8}\left(b_{i 1}(v) \xi_{1}+\cdots+b_{i 4}(v) \xi_{4}\right)
$$

branched along the eight hyperplanes $M_{b_{1}(v)}, \ldots, M_{b_{8}(v)}$. Here the equation is a weighted homogeneous equation. (The weight of $\eta$ is four.) We set $\Sigma=\left\{\tau_{1}, \ldots, \tau_{8}\right\} \subset p^{-1}(0)$. For a point $t \in \tilde{\mathcal{C}}-\Sigma$, the inverse image $\pi^{-1}\left(\gamma_{t}\right)$ has exactly two irreducible components. These irreducible components defines an etale double covering $\mathcal{C}^{\# 0} \rightarrow \tilde{\mathcal{C}}-\Sigma$. By the purity of branch locus, we have an etale double covering

$$
\mathcal{C}^{\#} \rightarrow \tilde{\mathcal{C}}
$$

extending the above covering.

Let $\mathcal{U}^{\# 0} \subset \mathcal{C}^{\# 0} \times_{\Delta(v)} \mathcal{X}$ be the universal family of rational curves in $\mathcal{X}$ parameterized by $\mathcal{C}^{\# 0}$ and $\mathcal{U}^{\#}$ the closure of $\mathcal{U}^{\# 0}$ in $\mathcal{C}^{\#} \times_{\Delta(v)} \mathcal{X}$.

Proposition 4.4. The covering 4.5 is a union of two copies $\tilde{\mathcal{C}}^{(1)}$ and $\tilde{\mathcal{C}}^{(2)}$ of $\tilde{\mathcal{C}}$

Proof. To prove the proposition, it is enough to show the fiber of the covering (4.5) at $v=0$ is a union of two copies of $\tilde{\mathcal{C}}_{0}$. The equation of the fiber of $\mathcal{X}$ 
and $\tilde{\mathcal{C}}$ at $v=0$ is

$$
\begin{aligned}
& \mathcal{\mathcal { X }}_{0}: \eta^{2}=\prod_{i=1}^{8}\left(\lambda_{i}^{3} \xi_{1}+\lambda_{i}^{2} \xi_{2}+\lambda_{i} \xi_{3}+\xi_{4}\right), \text { and } \\
& \tilde{\mathcal{C}}_{0}: y^{2}=\prod_{i=1}^{8}\left(x-\lambda_{i}\right) .
\end{aligned}
$$

By restricting the equation (4.6) to the curve $\gamma_{x}$ given in (4.2), we have

$$
\eta^{2}=\prod_{i=1}^{8}\left\{\left(w-\lambda_{i}\right)^{2}\left(x-\lambda_{i}\right)\right\} .
$$

Therefore the rational curve $\gamma_{x}$ can be lifted to a rational curve $\tilde{\gamma}_{x, y}^{(1)}$ in $\mathcal{X}_{0}$ defined by

$$
\begin{aligned}
& \left\{\left(\eta: \xi_{1}: \xi_{2}: \xi_{3}: \xi_{4}\right)=\left(y \prod_{i=1}^{8}\left(w-\lambda_{i}\right):-1: 2 w+x:-\left(w^{2}+2 x w\right): w^{2} x\right)\right. \\
& \left.\in \mathbf{P}(2,1,1,1) \mid w \in \mathbf{P}^{1}\right\} .
\end{aligned}
$$

Since the family $\left\{\tilde{\gamma}_{x, y}^{(1)}\right\}$ of rational curves is parameterized by $(x, y) \in \tilde{\mathcal{C}}_{0}$, we have a morphism $\tilde{\mathcal{C}_{0}} \rightarrow \mathcal{C}_{0}^{\#}$. Therefore the covering $\mathcal{C}_{0}^{\#} \rightarrow \tilde{\mathcal{C}}_{0}$ is a union of two copies $\tilde{\mathcal{C}}_{0}^{(1)}$ and $\tilde{\mathcal{C}}_{0}^{(2)}$ of $\tilde{\mathcal{C}}_{0}$.

Definition 4.5. Let $\mathcal{U}$ be the inverse image of $\mathcal{U}^{\#}$ by the open immersion $\tilde{\mathcal{C}} \times \mathcal{X} \simeq \tilde{\mathcal{C}}^{(1)} \times \mathcal{X} \rightarrow \tilde{\mathcal{C}}^{\#} \times \mathcal{X}$.

\section{Algebraic CORRespondence}

Let $U \subset C \times X$ be the family of rational curves on $X$ parameterized by $C$ defined in Definition 3.5. By using the cycle class $c l(U) \in H^{4}(C \times X, \mathbf{Q})(2)$, we have the following diagram of homomorphisms:

$$
\begin{gathered}
H^{1}(C \times X, \mathbf{Q}) \stackrel{c l(U)}{\rightarrow} H^{5}(C \times X, \mathbf{Q})(2) \stackrel{p r_{2 *}^{*}}{\rightarrow} H^{3}(X, \mathbf{Q})(1) \\
H^{1}(C, \mathbf{Q})
\end{gathered}
$$

Here $V(i)$ denotes the Tate twist of $V$. The composite homomorphism

$$
H^{1}(C, \mathbf{Q}) \rightarrow H^{3}(X, \mathbf{Q})(1) .
$$

is called the cylinder map for $U$. We consider the family $\mathcal{U}$ of the universal family over $\Delta(v)$ :

$$
\begin{array}{cccc}
\mathcal{U} \subset \tilde{\mathcal{C}} \times_{\Delta(v)} \mathcal{X} & \stackrel{p r_{2}}{\rightarrow} & \mathcal{X} \\
p r_{1} \downarrow & & \downarrow \Xi \\
\tilde{\mathcal{C}} & & \stackrel{p}{\rightarrow} & \Delta(v)
\end{array}
$$


Then we have a homomorphism $c y l$ of local systems induced by the family of algebraic correspondences $\mathcal{U}$ :

$$
c y l: R^{1} p_{*} \mathbf{Q} \rightarrow R^{3} \Xi_{*} \mathbf{Q}(1)
$$

Theorem 5.1. The homomorphism (5.2) is injective.

By specialization argument, we have the following corollary.

Corollary 5.2. The homomorphism (5.1) is injective.

Proof of Theorem 5.1. To prove the injectivity of the map (5.2), it is enough to show the injectivity for the fiber of (5.2) at $v=0$. In this case, the homomorphism cyl is described by the homomorphism $\varphi$ defined in \$1. By the the isomorphism (1.2), we have

$$
\left(\tilde{\mathcal{C}}_{0} \times \tilde{\mathcal{C}}_{0} \times \tilde{\mathcal{C}}_{0}\right) / G \simeq \mathcal{X}_{0}
$$

where the finite group $G$ is defined in $\oiint$, Let $(x, y)$ be a point in $\tilde{\mathcal{C}}_{0}$. By the equation (4.7), the rational curve $\tilde{\gamma}_{x, y}^{(1)}$ in $\mathcal{X}_{0}$ defined by (4.7) is equal to the image of the map $I_{x, y}$ defined by

$$
I_{x, y}: \tilde{\mathcal{C}}_{0} \rightarrow \mathcal{X}_{0}:\left(x_{1}, y_{1}\right) \mapsto \varphi\left(\left(x_{1}, y_{1}\right),\left(x_{1},-y_{1}\right),(x, y)\right) .
$$

Therefore the universal family $\mathcal{U} \subset \tilde{\mathcal{C}}_{0} \times \mathcal{X}_{0}$ is equal to the image of the map

$$
\tilde{\mathcal{C}}_{0} \times \tilde{\mathcal{C}}_{0} \longrightarrow \tilde{\mathcal{C}}_{0} \times \mathcal{X}_{0}:\left(\left(x_{1}, y_{1}\right),(x, y)\right) \mapsto\left((x, y), I_{x, y}\left(x_{1}, y_{1}\right)\right)
$$

Using this description of the universal family, the fiber $H^{1}\left(\tilde{\mathcal{C}}_{0}, \mathbf{Q}\right) \rightarrow H^{3}\left(\mathcal{X}_{0}, \mathbf{Q}\right)(1)$ of the homomorphism (5.2) at $v=0$ is identified with the map

$$
H^{1}\left(\tilde{\mathcal{C}}_{0}, \mathbf{Q}\right) \rightarrow \bigwedge^{3} H^{1}\left(\tilde{\mathcal{C}}_{0}, \mathbf{Q}\right)(1) \simeq H^{3}\left(\mathcal{X}_{0}, \mathbf{Q}\right)(1)
$$

given by $a_{1} \mapsto a_{1} \wedge \phi$, where $\phi$ corresponds to the polarization of $H^{1}\left(\tilde{\mathcal{C}_{0}}, \mathbf{Q}\right)$. Therefore it is injective.

\section{Cokernel of CYLINDER MAP AND INFINITESIMAL VARIATION OF MIXED HODGE STRUCTURE}

6.1. Hodge structures of double coverings and Jacobian rings. Let $M$ be the moduli space of genus three curves with level $2 N$ structures $(N \geq 2)$ and $p: \mathcal{C} \rightarrow M$ be the universal curve over $M$. Let $M_{h e}$ be the hyperelliptic locus of $M$ and the complement $M \backslash M_{h e}$ is denoted by $M^{0}$. Then the restriction $p^{0}: \mathcal{C}^{0}=p^{-1}\left(M^{0}\right) \rightarrow M^{0}$ is a family of plane quartic curves with level $2 N$ structures. By choosing one of the family of even theta divisors $\Theta$, we get a family

$$
\mathcal{B}^{0} \subset \mathbf{P}\left(p_{*}^{0} \mathcal{O}\left(K_{\mathcal{C}^{0} / M^{0}}+\Theta\right)^{*}\right) \rightarrow M^{0}
$$

of Cayley octads. The closure of $\mathcal{B}^{0}$ in $\mathbf{P}\left(p_{*} \mathcal{O}\left(K_{\mathcal{C} / M}+\Theta\right)^{*}\right)$ is denoted by $\mathcal{B}$. Then $\mathcal{B}$ is a disjoint union of eight copies of trivial covering of $M$. By taking an etale covering $\theta: \tilde{M} \rightarrow M$ of $M$, we can choose a double covering $\tilde{\Xi}: \tilde{\mathcal{X}} \rightarrow \tilde{M}$ of $\theta^{*} \mathbf{P}\left(p_{*} \mathcal{O}\left(K_{\mathcal{C} / M}+\Theta\right)\right) \rightarrow \tilde{M}$ branching along the family of dual hyperplanes corresponding to $\theta^{*} \mathcal{B}$. Moreover, by Theorem 5.1, we have 
an injective morphism of local systems $\theta^{*} R^{1} p_{*} \mathbf{Q}(-1) \rightarrow R^{3} \tilde{\Xi}_{*} \mathbf{Q}$ by replacing $\tilde{M}$ by its etale covering, if necessary. Since the covering transformation group $\tilde{\mathcal{X}} \rightarrow \theta^{*} \mathbf{P}\left(p_{*} \mathcal{O}\left(K_{\mathcal{C} / M}+\Theta\right)\right)$ acts as $(-1)$-multiplication on $R^{3} \tilde{\Xi}_{*} \mathbf{Q}(-1)$, the descent data for $\theta^{*} R^{1} p_{*} \mathbf{Q}(-1)$ gives rise to a descent data of $\tilde{\mathcal{X}}$. The descended variety $\Xi: \mathcal{X} \rightarrow M$ becomes a double covering of $\mathbf{P}\left(p_{*} \mathcal{O}\left(K_{\mathcal{C} / M}+\Theta\right)\right)$, which is called the universal family of double coverings.

Let $\mathcal{C}_{h e}$ be the restriction of $\mathcal{C}$ to the hyperelliptic locus $M_{h e}$. Since the third higher direct image sheaf $R^{3} j_{*} \mathbf{Q}$ of the relative Jacobian variety $j$ : $J\left(\mathcal{C}_{h e} / M_{h e}\right) \rightarrow M_{h e}$ is isomorphic to $\bigwedge^{3} R^{1} j_{*} \mathbf{Q}$, the restriction of the cokernel $\operatorname{Coker}(c y l)$ of

$$
\text { cyl : } R^{1} p_{*} \mathbf{Q}(-1) \rightarrow R^{3} \Xi_{*} \mathbf{Q}
$$

to the hyperelliptic locus $M_{h e}$ is isomorphic to the primitive part of the third higher direct image sheaf

$$
R^{3} j_{*, p r i m} \mathbf{Q}=\operatorname{Coker}\left(R^{1} j_{*} \mathbf{Q}(-1) \rightarrow R^{3} j_{*} \mathbf{Q}\right) .
$$

In this section, we prove that there does not exist a polarized abelian scheme $a: \mathcal{A} \rightarrow M$ whose primitive part $R^{3} a_{*, \operatorname{prim}} \mathbf{Q}$ is isomorphic to $\operatorname{Coker}(c y l)$.

We recall computations of the infinitesimal variations of Hodge structure of double coverings branched along normal crossing eight hyperplanes using Jacobian rings. Let $\tilde{b}_{1}=\left(b_{11}, \ldots, b_{41}\right), \ldots, \tilde{b}_{8}=\left(b_{18}, \ldots, b_{48}\right)$ be non-zero elements in $V$ such that such that the union $\cup_{i} H_{i}$ of the dual hyperplanes $H_{i}$ of $b_{i}=\left(b_{1 i}: \cdots: b_{4 i}\right) \in \mathbf{P}(V)$ is normal crossing. We normalize $\mathbf{B}=$ $\left({ }^{t} \tilde{b}_{1}, \ldots,{ }^{t} \tilde{b}_{8}\right)$ as follows :

$$
\mathbf{B}=\left(b_{i j}\right)_{i j}=\left(\begin{array}{cccccccc}
1 & 0 & 0 & 0 & 1 & 1 & 1 & 1 \\
0 & 1 & 0 & 0 & 1 & s_{11} & s_{12} & s_{13} \\
0 & 0 & 1 & 0 & 1 & s_{21} & s_{22} & s_{23} \\
0 & 0 & 0 & 1 & 1 & s_{31} & s_{32} & s_{33}
\end{array}\right) .
$$

We consider the covering $\widetilde{X}$ of $\mathbf{P}\left(V^{*}\right)=\left\{\left(\xi_{1}: \xi_{2}: \xi_{3}: \xi_{4}\right)\right\}$ in a weighted projective space defined by

$$
\eta_{i}^{2}=b_{1 i} \xi_{1}+\cdots+b_{4 i} \xi_{4} . \quad(i=1, \ldots, 8)
$$

Here the weight of $\eta_{i}$ is $\frac{1}{2}$ for $i=1, \ldots, 8$. Then $\widetilde{X}$ is a complete intersection of Fermat type quadrics in $\left.\mathbf{P}(W)=\left\{\eta_{1}: \cdots: \eta_{8}\right)\right\}$ defined by

$$
\begin{aligned}
& f_{1}=\eta_{5}^{2}-\left(\eta_{1}^{2}+\eta_{2}^{2}+\eta_{3}^{2}+\eta_{4}^{2}\right)=0 \\
& f_{2}=\eta_{6}^{2}-\left(\eta_{1}^{2}+s_{11} \eta_{2}^{2}+s_{21} \eta_{3}^{2}+s_{31} \eta_{4}^{2}\right)=0 \\
& f_{3}=\eta_{7}^{2}-\left(\eta_{1}^{2}+s_{12} \eta_{2}^{2}+s_{22} \eta_{3}^{2}+s_{32} \eta_{4}^{2}\right)=0 \\
& f_{4}=\eta_{8}^{2}-\left(\eta_{1}^{2}+s_{13} \eta_{2}^{2}+s_{23} \eta_{3}^{2}+s_{33} \eta_{4}^{2}\right)=0 .
\end{aligned}
$$

We set $F(\eta, q)=\sum_{j=1}^{4} q_{j} f_{j}(\eta)$. The Jacobian ideal $J(\tilde{X})$ of $\tilde{X}$ is an ideal of the bi-graded ring $\mathbf{C}\left[\eta_{1}, \ldots, \eta_{8}, q_{1}, \ldots, q_{4}\right]$ generated by $\frac{\partial F(\eta, q)}{\partial \eta_{i}}$ 
and $\frac{\partial F(\eta, q)}{\partial q_{j}}$. Here the bi-degrees of $\eta_{i}$ and $q_{i}$ are $(1,0)$ and $(0,1)$, respectively. We define the Jacobian $\operatorname{ring} R(\widetilde{X})$ of $\widetilde{X}$ by the quotient ring $\mathbf{C}\left[\eta_{1}, \ldots, \eta_{8}, q_{1}, \ldots, q_{4}\right] / J(\widetilde{X})$. Then we have the following proposition (see [T3]).

Proposition 6.1. (1) The Hodge component $H^{3-i, i}(\widetilde{X})$ is identified with $R(\widetilde{X})_{2 i, i}$ for $i=0,1,2,3$. The tangent space of the moduli space $H^{1}\left(\widetilde{X}, \Theta_{\widetilde{X}}\right)$ is identified with $R(\widetilde{X})_{2,1}$.

(2) Under the above identification, the Kodaira-Spencer map

$$
H^{1}\left(\widetilde{X}, \Theta_{\tilde{X}}\right) \otimes H^{3-i, i}(\widetilde{X}) \rightarrow H^{2-i, i+1}(\widetilde{X})
$$

is identified with the multiplication map of the Jacobian ring.

(3) There is an isomorphism $t: R(\widetilde{X})_{6,3} \stackrel{\widetilde{\Xi}}{\rightarrow} \mathbf{C}$ such that the cup product

$$
H^{3-i, i}(\widetilde{X}) \otimes H^{i, 3-i}(\widetilde{X}) \rightarrow H^{3,3}(\widetilde{X}) \simeq \mathbf{C}
$$

is identified with the following composite map

$$
R(\widetilde{X})_{2 i, i} \otimes R(\widetilde{X})_{2(3-i), 3-i} \rightarrow R(\widetilde{X})_{6,3} \stackrel{t}{\rightarrow} \mathbf{C} .
$$

The group $\tilde{G}=\mu_{2}{ }^{8}$ acts on the variety $\tilde{X}$ and the $\operatorname{ring} R(\tilde{X})$ by

$$
\left(\eta_{i}\right)_{i} \mapsto\left(m_{i} \eta_{i}\right)_{i} \text { for }\left(m_{i}\right) \in \tilde{G} .
$$

We set $G=\left\{\left(m_{i}\right)_{i} \in \widetilde{G} \mid \prod_{i} m_{i}=1\right\}$. Then the double covering $X$ is isomorphic to the quotient $X=\widetilde{X} / G$ of $\widetilde{X}$ by the action of $G$. Therefore the Hodge structure of $X$ is the invariant part of $H^{3}(\widetilde{X})$ under the action of $G$.

Proposition 6.2. Let $R(\tilde{X})^{\tilde{G}}$ be the fixed part of $R(\tilde{X})$. Under the identification in Proposition 6.1, we have

$$
H^{3-i, i}(X) \simeq R(\widetilde{X})_{2 i, i}^{\tilde{G}}
$$

for $i=0,1,2,3$. The tangent space of the moduli space coming from deformations of configurations of hyperplanes is identified with $R(\widetilde{X})_{2,1}^{\tilde{G}}$.

Proof. Let $\chi$ be the character of $\widetilde{G}$ defined by $\left(m_{i}\right)_{i} \mapsto \prod_{i} m_{i}$ and $\Omega$ be the differential form defined by

$$
\Omega=\left(\sum_{i=1}^{8}(-1)^{i} \eta_{i} \wedge \bigwedge_{j \neq i} d \eta_{j}\right) \wedge\left(\sum_{k=1}^{4}(-1)^{k} q_{k} \wedge \bigwedge_{j \neq k} d q_{j}\right)
$$

Recall that the isomorphism $R(\tilde{X})_{2 i, i} \simeq H^{3-i, i}(X)$ is obtained by the composite

$$
\begin{array}{cccc}
R(\widetilde{X})_{2 i, i} & \rightarrow & H^{6-i, 3+i}\left(\mathbf{P}^{7} \times \mathbf{P}^{3}-\{F(\eta, q)=0\}\right) & \simeq H^{3-i, i}(X) \\
f(\eta, q) & \mapsto & \frac{f(\eta, q) \Omega}{F(\eta, q)^{4+i}}
\end{array}
$$

Then the group $\widetilde{G}$ acts of on $\Omega$ and $H^{3}(X)$ via the character $\chi$. Therefore the action of $\widetilde{G}$ on $f$ is trivial. 
The invariant part of $R(\tilde{X})$ under the action of $\tilde{G}$ is isomorphic to $\bar{R}=$ $\mathbf{C}\left[u_{1}, \ldots, u_{8}, q_{1}, \ldots, q_{4}\right] / \bar{J}$, where $\eta_{i}^{2}=u_{i}$ and $\bar{J}$ is generated by

$$
\begin{aligned}
& u_{5}-\left(u_{1}+u_{2}+u_{3}+u_{4}\right), \quad u_{6}-\left(u_{1}+s_{11} u_{2}+s_{21} u_{3}+s_{31} u_{4}\right), \\
& u_{7}-\left(u_{1}+s_{12} u_{2}+s_{22} u_{3}+s_{32} u_{4}\right), \quad u_{8}-\left(u_{1}+s_{13} u_{2}+s_{23} u_{3}+s_{33} u_{4}\right), \\
& u_{5} q_{1}, \quad u_{6} q_{2}, \quad u_{7} q_{3}, \quad u_{8} q_{4}, \\
& u_{1}\left(q_{1}+q_{2}+q_{3}+q_{4}\right), \quad u_{2}\left(q_{1}+s_{11} q_{2}+s_{12} q_{3}+s_{13} q_{4}\right), \\
& u_{3}\left(q_{1}+s_{21} q_{2}+s_{22} q_{3}+s_{23} q_{4}\right), \quad u_{4}\left(q_{1}+s_{31} q_{2}+s_{32} q_{3}+s_{33} q_{4}\right) .
\end{aligned}
$$

By eliminating $u_{1}, u_{2}, u_{3}$ and $u_{4}$, the ring $\bar{R}$ is isomorphic to the quotient of $\mathbf{C}\left[u_{1}, \ldots, u_{4}, q_{1}, \ldots, q_{4}\right]$ by the ideal $J$ generated by

$$
\begin{aligned}
& \left(u_{1}+u_{2}+u_{3}+u_{4}\right) q_{1}, \quad\left(u_{1}+s_{11} u_{2}+s_{21} u_{3}+s_{31} u_{4}\right) q_{2}, \\
& \left(u_{1}+s_{12} u_{2}+s_{22} u_{3}+s_{32} u_{4}\right) q_{3}, \quad\left(u_{1}+s_{13} u_{2}+s_{23} u_{3}+s_{33} u_{4}\right) q_{4}, \\
& u_{1}\left(q_{1}+q_{2}+q_{3}+q_{4}\right), \quad u_{2}\left(q_{1}+s_{11} q_{2}+s_{12} q_{3}+s_{13} q_{4}\right), \\
& u_{3}\left(q_{1}+s_{21} q_{2}+s_{22} q_{3}+s_{23} q_{4}\right), \quad u_{4}\left(q_{1}+s_{31} q_{2}+s_{32} q_{3}+s_{33} q_{4}\right),
\end{aligned}
$$

We consider a matrix $\mathbf{B}$ as in (6.1) such that $B=\left\{b_{1}, \ldots, b_{8}\right\}$ form a regular Cayley octad. In this case, $s_{13}, s_{23}, s_{33}$ is determined from $s_{11}, s_{21}, s_{31}, s_{12}, s_{22}, s_{32}$ as follows. We solve the linear equation

$$
\left(\begin{array}{ccc}
1 & 1 & 1 \\
s_{11} & s_{21} & s_{31} \\
s_{12} & s_{22} & s_{32}
\end{array}\right)\left(\begin{array}{l}
\alpha_{1} \\
\alpha_{2} \\
\alpha_{3}
\end{array}\right)=\left(\begin{array}{l}
1 \\
1 \\
1
\end{array}\right), \quad\left(\begin{array}{ccc}
s_{11} & s_{21} & s_{31} \\
s_{12} & s_{22} & s_{32} \\
s_{11} s_{12} & s_{21} s_{22} & s_{31} s_{32}
\end{array}\right)\left(\begin{array}{l}
\beta_{1} \\
\beta_{2} \\
\beta_{3}
\end{array}\right)=\left(\begin{array}{l}
1 \\
1 \\
1
\end{array}\right)
$$

on $\alpha_{1}, \alpha_{2}, \alpha_{3}, \beta_{1}, \beta_{2}, \beta_{3}$. Then $s_{13}, s_{23}, s_{33}$ are obtained by

$$
s_{13}=\frac{\alpha_{1}}{\beta_{1}}, \quad s_{23}=\frac{\alpha_{2}}{\beta_{2}}, \quad s_{33}=\frac{\alpha_{3}}{\beta_{3}} .
$$

By substituting $s_{13}, s_{23}, s_{33}$ by the rational function of $s_{11}, \ldots, s_{32}$, the coefficient of $F(u, p)$ is a rational function on $s_{11}, \ldots, s_{32}$. We set

$$
\begin{aligned}
\tau_{1}=\frac{\partial F}{\partial s_{11}}, & \tau_{2}=\frac{\partial F}{\partial s_{21}}, & \tau_{3}=\frac{\partial F}{\partial s_{31}} \\
\tau_{4}=\frac{\partial F}{\partial s_{12}}, & \tau_{5}=\frac{\partial F}{\partial s_{22}}, & \tau_{6}=\frac{\partial F}{\partial s_{32}} .
\end{aligned}
$$

Let $T$ be the linear span of the set $\left\{\tau_{i}\right\}_{i=1, \ldots, 6}$ in $\bar{R}$. Then the vector space $T$ is the tangent space of the moduli space of Cayley octads at $B$.

\subsection{Infinitesimal variations of Hodge structure for abelian schemes.} In this section, we use the same notations $C, X$ and $B$ of the last subsection. The point in $M$ corresponding to $C$ is denoted by $P$. We recall the definition of infinitesimal variations of polarized Hodge structure.

Definition 6.3 (Infinitesimal variation of polarized Hodge structure [CG]) A triple $\left(\oplus H^{i, j}, \theta,(),\right)$ consisting of

(1) a vector space $\oplus_{i+j=q} H^{i j}$ with a linear map $\theta: H^{i, j} \rightarrow H^{i-1, j+1}$, and

(2) a system of $(-1)^{q}$-symmetric perfect pairing $():, H^{i, j} \otimes H^{j, i} \rightarrow \mathbf{C}$ 
is called an infinitesimal variation of polarized Hodge structure of weight $q$ if $(\theta(a), b)+(a, \theta(b))=0$.

To a variation of Hodge structures $\mathcal{H}$ of weight $q$ over $M$ and a tangent vector at a point $P$ in $M$, we can naturally associate an infinitesimal variation of polarized Hodge structure on the direct product $\oplus_{i+j=q} \mathcal{H}_{P}^{i, j}$ of Hodge components of the fiber $\mathcal{H}_{P}$. The variation of Hodge structures $R^{3} \Xi_{*} \mathbf{Q}$ and a tangent vector $\theta$ at $P$ give rise to the following infinitesimal variation of polarized Hodge structure.

$$
\theta_{X}: H^{3-i, i}(X) \rightarrow H^{2-i, i+1}(X) .
$$

Similarly, the variation of Hodge structures $R^{1} p_{*} \mathbf{Q}$ gives rise to the following infinitesimal variation of polarized Hodge structure.

$$
\theta_{C}: H^{1,0}(C) \rightarrow H^{0,1}(C) .
$$

Since the homomorphism (5.2) comes form a family of algebraic correspondences, the two infinitesimal variations $\theta_{C}, \theta_{X}$ are compatible. Therefore we have the following commutative diagram.

$$
\begin{array}{cccc}
H^{1,0}(C) & \underline{\theta}_{G} & H^{0,1}(C) \\
\text { cyl } & & \downarrow c y l \\
H^{2,1}(X) & \rightarrow & H^{1,2}(X)
\end{array}
$$

The vertical map $c y l$ is injective by Theorem 5.1. The cokernel of the map cyl is an infinitesimal variation of polarized Hodge structure, which is denoted by $\left(H_{\text {prim }}^{3}(X), \theta_{X}\right)$.

Let $H_{\text {prim }}^{2,1}(X)$ and $H_{\text {prim }}^{1,2}(X)$ be the orthogonal complements of the image of $H^{0,1}(C)$ and $H^{1,0}(C)$, respectively. Then the restriction of the map $\theta_{X}$ to $H^{3,0} \oplus H_{\text {prim }}^{2,1} \oplus H_{\text {prim }}^{1,2} \oplus H^{0,3}$ is an infinitesimal variation of polarized Hodge structure and it isomorphic to $\left(H_{\text {prim }}^{3}(X), \theta_{X}\right)$. Then the map

$$
T M_{P} \rightarrow \operatorname{Hom}\left(H^{3,0}(X), H_{\text {prim }}^{1,2}(X)\right)
$$

defined by $\theta \mapsto \theta_{X} \circ \theta_{X}$ is a $\operatorname{Hom}\left(H^{3,0}\left(X_{p}\right), H_{\text {prim }}^{1,2}(X)\right)$-valued quadratic form on $T M_{P}$, which is denoted as $\mathcal{Q}_{X}$.

We consider infinitesimal variations of Hodge structure arising from weight one variations. Let $H^{1,0}, H^{0,1}$ be 3 -dimensional $\mathbf{C}$-vector spaces and (, ) be a non-degenerate pairing $H^{1,0} \otimes H^{0,1} \rightarrow \mathbf{C}$. Using the isomorphism $\left(H^{1,0}\right)^{*} \stackrel{\iota}{\rightarrow}$ $H^{0,1}$ and $\left(H^{0,1}\right)^{*} \stackrel{\iota}{\rightarrow} H^{1,0}$ obtained by this pairing, we have the following isomorphism :

$$
\operatorname{Hom}\left(H^{0,1}, H^{1,0}\right)=\operatorname{Hom}\left(\left(H^{1,0}\right)^{*},\left(H^{0,1}\right)^{*}\right) \stackrel{\iota}{\rightarrow} \operatorname{Hom}\left(H^{0,1}, H^{1,0}\right) .
$$

The image of $\theta$ under the above isomorphism is denoted by ${ }^{t} \theta$. For a linear map $\theta \in H o m\left(H^{1,0}, H^{0,1}\right)$, the triple $(\mathbf{H}, \theta)=\left(H^{1,0} \oplus H^{0,1}, \theta: H^{1,0} \rightarrow\right.$ $\left.H^{0,1},(),\right)$ is an infinitesimal variation of Hodge structure if and only if $\theta={ }^{t} \theta$. We set

$$
T=\left\{\theta \in \operatorname{Hom}\left(H^{1,0}, H^{0,1}\right) \mid \theta={ }^{t} \theta\right\} .
$$


The third exterior product $\left(\bigwedge^{3} \mathbf{H}, \theta^{(3)}\right)$

$$
\wedge^{3} H^{1,0} \stackrel{\theta^{(3)}}{\rightarrow} \wedge^{2} H^{1,0} \otimes H^{0,1} \stackrel{\theta^{(3)}}{\rightarrow} H^{1,0} \otimes \wedge^{2} H^{0,1} \stackrel{\theta^{(3)}}{\rightarrow} \wedge^{3} H^{0,1}
$$

of $(\mathbf{H}, \theta)$ is given by the formula

$$
\theta^{(3)}\left(a_{1} \wedge a_{2} \wedge a_{3}\right)=\theta\left(a_{1}\right) \wedge a_{2} \wedge a_{3}+a_{1} \wedge \theta\left(a_{2}\right) \wedge a_{3}+a_{1} \wedge a_{2} \wedge \theta\left(a_{3}\right)
$$

for $a_{1}, a_{2}, a_{3} \in H^{0,1} \oplus H^{1,0}$. Let $\phi$ be the element in $\bigwedge^{2} \mathbf{H}$ corresponding to the identity map of $\operatorname{Hom}\left(H^{0,1}, H^{0,1}\right)$ via the following isomorphism:

$$
\operatorname{Hom}\left(H^{0,1}, H^{0,1}\right) \simeq\left(H^{0,1}\right)^{*} \otimes H^{0,1} \simeq H^{1,0} \otimes H^{0,1} \subset \bigwedge^{2} \mathbf{H} .
$$

We set $H_{\text {prim }}^{2,1}$ and $H_{\text {prim }}^{1,2}$ the orthogonal complements of $\phi \wedge H^{0.1} \subset H^{1,2}$ and $\phi \wedge H^{1.0} \subset H^{2,1}$, respectively. Then we have an infinitesimal variation of Hodge structure

$$
\left(\bigwedge^{3} \mathbf{H}\right)_{\text {prim }}=\left(\bigwedge^{3} H^{1,0} \oplus H_{\text {prim }}^{2,1} \oplus H_{\text {prim }}^{1,2} \oplus \bigwedge^{3} H^{0,1}, \theta^{(3)},(,)\right) .
$$

which is isomorphic to $\operatorname{Coker}\left(\mathbf{H} \rightarrow \bigwedge^{3} \mathbf{H}\right)$. Then the map

$$
\mathcal{Q}: T \rightarrow \operatorname{Hom}\left(\bigwedge^{3} H^{1,0}, H_{\text {prim }}^{1,2}\right): \theta \mapsto \theta^{(3)} \circ \theta^{(3)}
$$

is a $\operatorname{Hom}\left(\bigwedge^{3} H^{1,0}, H_{\text {prim }}^{1,2}\right)$-valued quadratic form on $T$. We have the following proposition.

Proposition 6.4. There exists an non-zero element $\theta \in T$ such that $\mathcal{Q}(\theta)=$ 0 .

Proof. Let $e_{1}, e_{2}, e_{3}$ be a basis of $H^{1,0}$ and $f_{1}, f_{2}, f_{3}$ be the dual base of $H^{0,1}$. Let $\theta \in H o m\left(H^{1,0}, H^{0,1}\right)$ be the map defined by $\theta\left(e_{1}\right)=f_{1}, \theta\left(e_{2}\right)=\theta\left(e_{3}\right)=0$. Then $\theta \in T$ and we have

$$
\left(\theta^{(3)} \circ \theta^{(3)}\right)\left(e_{1} \wedge e_{2} \wedge e_{3}\right)=\theta^{(3)}\left(f_{1} \wedge e_{2} \wedge e_{3}\right)=0
$$

Proposition 6.5. There exists a regular Cayley octad $B=\left\{b_{1}, \ldots, b_{8}\right\}$ with the following property.

(1) The value $\mathcal{Q}(\theta)_{X}$ of $\mathcal{Q}_{X}$ at $\theta$ is non-zero for all $\theta \neq 0$.

(2) The map $T M_{P} \rightarrow \operatorname{Hom}\left(H^{3,0}(X), H^{2,1}(X)\right)$ is an isomorphism.

We give an explicit example of Cayley octad with the above properties in the next section. By Proposition 6.4 together with Proposition 6.5, we have the following theorem.

Theorem 6.6. There exists no polarized abelian scheme $a: \mathcal{A} \rightarrow M$ of relative dimension three, whose primitive part $R^{3} a_{\text {prim, }} \mathbf{Q}$ of $R^{3} a_{*} \mathbf{Q}$ is isomorphic to Coker(cyl) as variations of Hodge structures. 
Proof. Assume that there exists an abelian scheme $a: \mathcal{A} \rightarrow M$ such that $R^{3} a_{*, \text { prim }} \mathbf{Q} \simeq \operatorname{Coker}($ cyl $)$. Let $P^{\prime} \in M_{\text {et }}$ be a point corresponding to a hyperelliptic curve. Then the image of the fundamental group $\pi_{1}\left(M, P^{\prime}\right)$ under the monodromy representation on $\operatorname{Aut}\left(\left(R^{3} a_{*, \text { prim }} \mathbf{Q}\right)_{P^{\prime}}\right)$ contains the image of the fundamental group $\pi_{1}\left(M_{h e}, P^{\prime}\right)$ of $M_{h e}$, which is finite index in $S p(3, \mathbf{Z})$ by [TS]. Therefore there is at most one polarization on $R^{3} a_{*, p r i m} \mathbf{Q}$ and Coker $(c y l)$ and the isomorphism of variations of Hodge structures between $R^{3} a_{\text {prim, } *} \mathbf{Q}$ and Coker $(c y l)$ induces an isomorphisms of infinitesimal variation of polarized Hodge structure for any $\theta \in T M_{P}$ at any $P \in M$.

We consider the infinitesimal variation of polarized Hodge structure at the point $P$ corresponding to the Cayley octad satisfying the condition of Proposition 6.5. By the condition (2) and Proposition 6.4, there exists non-zero tangent vector $\theta \in M T_{P}$ such that $\theta^{(3)} \circ \theta^{(3)}=0$ in $\operatorname{Hom}\left(H^{3,0}\left(\mathcal{A}_{P}\right), H_{\text {prim }}^{2,1}\left(\mathcal{A}_{P}\right)\right)$. But this implies $\theta_{X} \circ \theta_{X}=0$, which contradicts to the condition (1) of Proposition 6.5.

\section{Example Satisfying the Condition in Proposition 6.5}

We compute examples of the Jacobian ring using Gröbner base under the computer program Maple. We show that there exists a Cayley octad satisfying the condition in Proposition 6.5. Under the notation of the last section, we set

$$
s_{11}=-1, s_{21}=3, s_{31}=4, s_{12}=-3, s_{22}=2, s_{32}=3 .
$$

Then $s_{13}=\frac{33}{5}, s_{23}=22, s_{33}=99$.

We identify the space $H^{2,1}\left(X_{p}\right)$ with the degree $(2,2)$ part of $\mathbf{Q}\left[u_{1}, \ldots, u_{4}, q_{1}, \ldots, q_{4}\right] / J$. Using graded reverse lexicographic order of $u_{4}, u_{3}, u_{2}, u_{1}, q_{4}, q_{3}, q_{2}, q_{1}, H^{2,1}\left(X_{p}\right)$ is isomorphic to the vector spanned by the class of

$$
\begin{array}{lll}
M_{1}=u_{2}^{2} p_{2}^{2}, & M_{2}=u_{2} u_{1} p_{2}^{2}, \quad M_{3}=u_{1}^{2} p_{2}^{2}, \quad M_{4}=u_{3}^{2} p_{1}^{2}, \quad M_{5}=u_{3} u_{2} p_{1}^{2} \\
M_{6}=u_{2}^{2} p_{1}^{2}, & M_{7}=u_{3} u_{1} p_{1}^{2}, \quad M_{8}=u_{2} u_{1} p_{1}^{2}, \quad M_{9}=u_{1}^{2} p_{1}^{2} .
\end{array}
$$

Therefore we have an isomorphism $r: H^{2,1}\left(X_{p}\right) \stackrel{\simeq}{\rightarrow} \mathbf{C} M_{1} \oplus \cdots \oplus \mathbf{C} M_{9}$. By a simple calculation, the tangent vectors $\tau_{1}, \ldots, \tau_{6}$ in (6.2) can be computed as

$$
\tau_{1}=q_{2} u_{2}+q_{4}\left(-6 u_{2}-\frac{73}{2} u_{3}-420 u_{4}\right)
$$

and so on. Let $\theta=w_{1} \tau_{1}+\cdots+w_{6} \tau_{6}$ be a linear combination of $\tau_{1}, \cdots \tau_{6}$. Then $r\left(\theta^{2}\right)$ is a $\mathbf{C}^{6}$-valued quadratic form on $w_{1}, \ldots, w_{6}$. The coefficients $f_{1}, \ldots, f_{9}$ 
of $M_{1}, \ldots, M_{9}$ are computed as

$$
\begin{aligned}
f_{1}= & -\frac{24757}{1240} w_{5}^{2}-\frac{1197}{155} w_{5} w_{6}+\frac{399}{62} w_{2} w_{6}+\frac{247}{124} w_{2} w_{4}+\frac{4351}{310} w_{2} w_{5} \\
& +\frac{3857}{1240} w_{3}^{2}-\frac{1463}{1488} w_{3} w_{4}+\frac{399}{62} w_{3} w_{5}-\frac{31787}{3720} w_{3} w_{6}+\frac{29735}{35712} w_{4}^{2} \\
& -\frac{209}{31} w_{4} w_{5}+\frac{15295}{8928} w_{4} w_{6}+\frac{133}{248} w_{1} w_{3}+\frac{95}{62} w_{1} w_{2}+\frac{12255}{992} w_{1}^{2} \\
& -\frac{20653}{2976} w_{1} w_{4}-\frac{95}{62} w_{2}^{2}-\frac{798}{155} w_{2} w_{3}+\frac{52535}{8928} w_{6}^{2}-\frac{2261}{1488} w_{1} w_{6} \\
& +\frac{323}{62} w_{1} w_{5},
\end{aligned}
$$

and so on. Then by using Gröbner basis, we conclude that

$$
\left\{\left(w_{1}, \ldots, w_{6}\right) \mid f_{1}\left(w_{1}, \ldots, w_{6}\right)=\cdots=f_{9}\left(w_{1}, \ldots, w_{6}\right)=0\right\}=\{(0, \ldots, 0)\} .
$$

Thus we have a regular Cayley octad $B$ satisfying the conditions of Proposition 6.5.

\section{REFERENCES}

[DO] I. Dolgachev and D. Ortland, Point sets in projective spaces and theta functions, Asterisque 165 (1988).

[CG] J. Carlson and P. Griffiths, Infinitesimal variation of Hodge structure and the global Torelli problem, in Journées de Géometrie Algébrique d'Anger, Sijthoff \& Noordhoff, (1980), p 51-76.

[CM] S. Cynk and C. Meyer, Geometry and arithmetic of certain double octic Calabi-Yau manifolds, Canad. Math. Bull. 48 (2005), no.2 , 180-194.

[GSZ] R. Gerkmann, M. Sheng and K. Zuo, Disproof of Modularity of Moduli Space of CY 3-folds of Double covers of $\mathbf{P}^{3}$ ramified along eight planes in General Positions, math.arXiv,0709.1051

[T1] T. Terasoma, Complete intersection of hypersurfaces.- Fermat case and quadratic case. Japanese J. of Math. 14-2 (1988) p.329-384.

[T2] T. Terasoma, Exponential Kummer covering and determinant of hypergeometric functions, Tokyo J. of Mathematics 132 (1990), p.213-235.

[T3] T. Terasoma, Weak global Torelli theorem for complete intersection of hypersurfaces. Ann. of Mathematics 132 (1990), p.213-235.

[TS] S. Tsuyumine, Thetanullwerte on a moduli space of curves and hyperelliptic loci. Math. Z. 207 (1991), no. 4, 539-568.

Graduate School of Mathematical Sicences, the University of Tokyo, 3-8-1 Komaba, Meguro, Tokyo, 153-8914, Tokyo, Japan 